\title{
Diffraction at a time grating in above-threshold ionization: The influence of the Coulomb potential
}

\author{
Diego G. Arbó, ${ }^{1,2}$ Kenichi L. Ishikawa, ${ }^{3}$ Klaus Schiessl,${ }^{4}$ Emil Persson, ${ }^{4}$ and Joachim Burgdörfer ${ }^{4}$ \\ ${ }^{1}$ Institute for Astronomy and Space Physics, IAFE (CONICET-UBA), CC 67, Suc. 28 (1428) Buenos Aires, Argentina \\ ${ }^{2}$ Department of Physics, FCEN, University of Buenos Aires, Argentina \\ ${ }^{3}$ Photon Science Center, Graduate School of Engineering, University of Tokyo, Hongo 7-3-1, Bunkyo-ku, Tokyo 113-8656, Japan \\ ${ }^{4}$ Institute for Theoretical Physics, Vienna University of Technology, Wiedner Hauptstraße 8-10/136, A-1040 Vienna, Austria, EU
}

(Received 28 July 2010; published 22 October 2010)

\begin{abstract}
We analyze the photoelectron emission spectrum in atomic above-threshold ionization by a linearly polarized short-laser pulse. Direct electrons can be characterized by both intracycle and intercycle interferences. The former results from the coherent superposition of two different electron trajectories released in the same optical cycle, whereas the latter is the consequence of the superposition of multiple trajectories released in different cycles. In the present article, a semiclassical analytical expression for the complete (both intracycle and intercycle) interference pattern is derived. We show that the recently proposed semiclassical description in terms of a diffraction process at a time grating remains qualitatively unchanged in the presence of the long-range Coulomb potential. The latter causes only a phase shift of the intracycle interference pattern. We verify the predictions of the semiclassical model by comparison with full three-dimensional (3D) time-dependent Schrödinger equation (TDSE) solutions. One key finding is that the subcycle interference structures originating from trajectories launched within a time interval of less than 1 femtosecond should be experimentally observable also in low-resolution spectra for longer multicycle pulses.
\end{abstract}

DOI: 10.1103/PhysRevA.82.043426

PACS number(s): $32.80 . \mathrm{Rm}, 32.80 . \mathrm{Fb}, 03.65 . \mathrm{Sq}$

\section{INTRODUCTION}

Above-threshold ionization and high-order harmonic generation (HHG) are highly nonlinear quantum-mechanical phenomena induced by intense laser pulses. The wavelength $\lambda$ dependence of the HHG yield has recently been studied extensively [1-4]. Superimposed on a smooth power-law dependence, Schiessl et al. [1,4] have found surprisingly strong and rapid oscillations on a fine $\lambda$ scale, which are due to quantum interference of many returning orbits and related to the channel closing $[5,6]$. Indeed, the period of the oscillation is unity in terms of the channel-closing number $R$

$$
R=\frac{I_{p}+U_{p}}{\omega},
$$

where $I_{p}, U_{p}$, and $\omega$ are the ionization potential, the ponderomotive energy of the electron in the laser field, and the carrier laser frequency, respectively. This observation naturally invites the question: Does high-intensity ionization yield also oscillate as a function of the laser wavelength or the channel-closing number $R$ ? According to the three-step model, photoelectrons can be classified into direct and rescattered electrons [7]. Electrons are emitted by tunneling through the potential barrier formed by the combination of the atomic potential and the external strong field. Tunneling occurs within each optical cycle predominantly around the maxima of the absolute value of the electric field. After detachment from the atom, direct electrons can escape without being strongly affected by the residual core potential. The classical cutoff energy for this process is $2 U_{p}$. After being accelerated back by the laser field, a small portion of electrons are rescattered by the parent ion and can achieve a kinetic energy $E$ of up to $10 U_{p}$. Similarly to HHG yield, Milošević et al. [8] found periodic enhancements in the intensity-dependent emission of rescattered electrons due to constructive interference of a large number of long quantum orbits. Martiny and Madsen [9] have observed interferences for ionization by circularly polarized short pulses, explained as the contribution of many frequencies of the power spectrum.

Classical trajectories that correspond to direct ionization $\left(E<2 U_{p}\right)$ are seemingly unrelated to HHG since they do not return close to the parent ion and, even if driven back, suffer only a distant collision with the core. Nevertheless, they are crucial in the formation of interference patterns in photoelectron spectra. A temporal double-slit interference pattern has recently been studied in near-single cycle pulses both experimentally $[10,11]$ and theoretically [12]. A time-energy analysis of above-threshold ionization has been recently presented [13]. Near threshold oscillations in angular distribution were explained as interferences of electron trajectories [14] and recently measured [15]. Diffraction fringes have been experimentally observed in photoionization of $\mathrm{He}$ atoms [11] and photodetachment in $\mathrm{F}^{-}$ions by femtosecond pulses for fixed frequency [16] and theoretically analyzed [17]. The interference pattern in multicycle photoelectron spectra can be identified as a diffraction pattern at a time grating composed of intracycle and intercycle interferences $[17,18]$. While the latter gives rise to the well-known above-threshold ionization (ATI) peaks, the former leads to a modulation of the ATI spectrum offering information on the subcycle ionization dynamics. This analysis was based on a one-dimensional (1D) semiclassical model closely following the "simple man's model" (SMM). Similar patterns were found in spectra of laser-assisted Auger decay whose gross structure of sidebands were explained as the interference between electrons emitted during one period [19].

In the present article we show that the analysis in terms of a time grating remains valid in the presence of long-range Coulomb forces. We gauge the semiclassical model by a comparison with the numerical results of the time-dependent distorted wave Coulomb-Volkov approximation (CVA) [20-24] and solutions of the full time-dependent Schrödinger 
equation (TDSE). Moreover, we present a systematic study of the dependence of intracycle and intercycle interferences on the length of the pulse. One key finding is that the observation of subcycle ionization dynamics should become accessible for longer multicycle pulses and, moreover, in low-resolution spectra provided the photoelectrons are observed along the axis of linear polarization. The dependence of the total ionization yield on $R$ (or equivalently, the wavelength of the driving field $\lambda$ ) will be analyzed, employing two-dimensional interferograms as a function of $E$ and $R$.

The article is organized as follows. In Sec. II we summarize the quantum-mechanical methods, the strong-field approximation (SFA), CVA, and the (exact) numerical solution of the full TDSE which serve as benchmarks for the semiclassical description. In Sec. III we expand on the previously presented semiclassical analysis [18] and show that long-range Coulomb effects leave the separation of intracycle and intercycle interferences and thus the interpretation of the interference pattern in terms of a diffraction at a time grating intact. In Sec. IV, we compare the photoelectron energy spectrum as a function of the laser wavelength calculated by these methods with the prediction of the semiclassical model and discuss similarities and differences. We show that for particular pulse shapes, intercycle interference can be suppressed completely preserving a pure intracycle interference pattern. We also investigate the wavelength dependence of the energy-integrated yield and find regular oscillations closely resembling those previously observed for HHG [1-4].

\section{BRIEF REVIEW OF QUANTUM METHODS}

In this section, we summarize approximate as well as exact numerical methods used to solve the Schrödinger equation of one-electron atoms subject to a strong laser pulse. These results will serve to probe the semiclassical description of interferences to be discussed in Sec. III. We consider an atom in the single active electron approximation interacting with a laser field $\vec{F}(t)$, linearly polarized along the $z$ direction. The Hamiltonian of the system in the length gauge is

$$
H=\frac{\vec{p}^{2}}{2}+V(r)+z F(t),
$$

where $V(r)$ is the atomic potential, $\vec{p}$ is the momentum, and $\vec{r}$ the position of the electron.

As a consequence of the interaction, the electron initially bound in the state $\left|\phi_{i}\right\rangle$ is emitted with momentum $\vec{k}$ and energy $E=k^{2} / 2$ into the final unperturbed state $\left|\phi_{f}\right\rangle$. The evolution of the electronic state $|\psi(t)\rangle$ is governed by the TDSE for the Hamiltonian of Eq. (2). The photoelectron momentum distributions can be calculated as

$$
\frac{d P}{d \vec{k}}=\left|T_{i f}\right|^{2},
$$

where $T_{i f}$ is the $T$-matrix element corresponding to the transition $\phi_{i} \rightarrow \phi_{f}$. The energy spectrum can be written as

$$
\frac{d P}{d E}=2 \pi \int_{-1}^{1} d(\cos \theta) \sqrt{2 E}\left|T_{i f}\right|^{2},
$$

where $\theta$ is the angle subtended by $\vec{k}$ and the direction of polarization.

\section{A. Time-dependent distorted wave theory}

We employ the time-dependent distorted wave theory in two variants: the CVA and the SFA. Within the time-dependent distorted wave theory [25], the transition amplitude in the post form is expressed as

$$
T_{i f}=-i \int_{-\infty}^{+\infty} d t\left\langle\chi_{f}^{-}(t)|z F(t)| \phi_{i}(t)\right\rangle,
$$

where $\chi_{f}^{-}(t)$ is the final distorted-wave function and the initial state $\phi_{i}(t)$ is an eigenstate of the atomic Hamiltonian without perturbation. If we choose the Hamiltonian of a free electron in the time-dependent electric field as the exit-channel distorted Hamiltonian [i.e., $\left.i \frac{\partial}{\partial t}\left|\chi_{f}^{-}(t)\right\rangle=\left(\frac{p^{2}}{2}+z F(t)\right)\left|\chi_{f}^{-}(t)\right\rangle\right]$ the solutions are the Volkov states [26]

$$
\chi_{\vec{k}}^{(V)-}(\vec{r}, t)=\frac{\exp [i(\vec{k}+\vec{A}) \vec{r}]}{(2 \pi)^{3 / 2}} \exp [i S(t)],
$$

where $S$ denotes the Volkov action

$$
S\left(t^{\prime}, t\right)=-\int_{t^{\prime}}^{t} d t^{\prime \prime}\left[\frac{\left[\vec{k}+\vec{A}\left(t^{\prime \prime}\right)\right]^{2}}{2}+I_{p}\right] .
$$

In Eq. (6), $S(t)$ must be understood as the action of Eq. (7) for the special case of $t^{\prime} \rightarrow-\infty$. In Eqs. (6) and (7), $\vec{A}(t)=$ $-\int_{-\infty}^{t} d t^{\prime} \vec{F}\left(t^{\prime}\right)$ is the vector potential of the laser field divided by the speed of light. Equation (5) together with Eq. (6) leads to the SFA transition matrix. Accordingly, the influence of the atomic core potential on the continuum state of the receding electron is neglected and therefore the momentum distribution is a constant of motion after the conclusion of the laser pulse. It is well known that the SFA fails to describe ionization for moderately weak fields as well as the slow electron yield even for strong fields [27].

An improved approximation is achieved by combining the atomic eigenstate of the continuum $\phi_{\vec{k}}^{-}$with the finalchannel wave function of Eq. (6). For a hydrogenic atom (i.e., $V(r)=-Z_{T} / r$ with $Z_{T}$ the nucleus charge) this results in the Coulomb-Volkov final state [28]

$$
\chi_{\vec{k}}^{(\mathrm{CV})-}(\vec{r}, t)=\chi_{\vec{k}}^{(V)-}(\vec{r}, t) \mathcal{D}_{C}\left(Z_{T}, \vec{k}, \vec{r}\right),
$$

where $\mathcal{D}_{C}\left(Z_{T}, \vec{k}, \vec{r}\right)=N_{T}^{-}(k){ }_{1} F_{1}\left(-i Z_{T} / k, 1,-i k r-i \vec{k} \vec{r}\right)$. The Coulomb normalization factor $N_{T}^{-}(k)=$ $\exp \left(\pi Z_{T} / 2 k\right) \Gamma\left(1+i Z_{T} / k\right)$ coincides with the value of the Coulomb wave function at the origin, and ${ }_{1} F_{1}$ denotes the confluent hypergeometric function. In the CVA, the simultaneous interactions of the released electron with the residual ionic core and the external field are taken into account nonperturbatively, yet approximately. Inserting Eq. (8) into Eq. (5) leads to the CVA, which can be evaluated in closed form [20,21]. From Eq. (8), the SFA can be derived as the limit of weak Coulomb potential, that is, $\chi_{\vec{k}}^{(\mathrm{CV})-} \rightarrow \chi_{\vec{k}}^{(V)-}$ of Eq. (6) as $Z_{T} \rightarrow 0$.

\section{B. Full solution of the three-dimensional TDSE}

The numerical solution of the TDSE is considered to be exact and will be used as an ultimate benchmark for assessing the reliability of the semiclassical model as well as the 
time-dependent distorted-wave (SFA and CVA). We solve the atomic TDSE

$$
i \frac{\partial}{\partial t}|\psi(\vec{r}, t)\rangle=H|\psi(\vec{r}, t)\rangle,
$$

where $H$ is the Hamiltonian of an atom subject to an external electric field given by Eq. (2). The TDSE is integrated on a finite grid by means of the pseudospectral method $[27,29]$. The $r$ coordinate is discretized using a finite-element discrete-variable method [30,31] with a nonuniform mesh point distribution allowing for an accurate description of the Coulomb singularity. Both the unbound as well as the bound parts of the wave function $|\psi(t)\rangle$ are accurately represented. For the present simulations, we are able to keep the entire wave function on the numerical grid such that no absorbing boundary conditions have to be invoked.

\section{SEMICLASSICAL MODEL}

In this section we present the semiclassical model for separating intracycle from intercycle interferences and extend this analysis to the long-range Coulomb interaction. We also investigate the dependence on the momentum of the emitted electron and the Keldysh parameter.

\section{A. Intracycle and intercycle interferences}

While a small fraction of photoelectrons undergoes rescattering by the remaining ion, we consider here direct photoelectrons (with energies $E \leqslant 2 U_{p}$ ) which dominate the total ionization yield. We closely follow the SMM [7,22-24] to deal with interference signatures within the SFA. A starting point is the saddle-point approximation of the SFA, which leads to a transition amplitude to the continuum state of the form [22]

$$
T_{i f}(\vec{k})=-\sum_{i=1}^{M} G\left[t_{r}^{(i)}, \vec{k}\right] e^{i S\left[t_{r}^{(i)}\right]} .
$$

Here $M$ is the number of classical trajectories reaching a given final momentum $\vec{k}$ and $G\left[t_{r}^{(i)}, \vec{k}\right]$ is the ionization amplitude

$$
G\left[t_{r}^{(i)}, \vec{k}\right]=\left[\frac{2 \pi i F\left[t_{r}^{(i)}\right]}{\left|\vec{k}+\vec{A}\left[t_{r}^{(i)}\right]\right|}\right]^{1 / 2} d^{*}\left(\vec{k}+\vec{A}\left[t_{r}^{(i)}\right]\right),
$$

where $d^{*}(\vec{v})$ is the dipole element of the bound-continuum transition. In Eq. (10), $S$ is given by the Volkov action [see Eq. (7)] [26]. Note that in line with the SMM the action does not contain contributions from the long-range Coulomb forces the ejected electron is subject to. We will return to this point in the following. Dropping the second argument in $S\left(t^{\prime}, t\right)$ for notational simplicity with the understanding that $t$ is the time at which the wave packet is projected onto observables (eventually $t \rightarrow \infty$ ), the release time $t_{r}^{(i)}$ of trajectory $i$ is determined by the saddle-point equation

$$
\left.\frac{\partial S\left(t^{\prime}\right)}{\partial t^{\prime}}\right|_{t^{\prime}=t_{r}^{(i)}}=\frac{\left[\vec{k}+\vec{A}\left[t_{r}^{(i)}\right]\right]^{2}}{2}+I_{p}=0 .
$$

Release times $t_{r}^{(i)}$ are complex since $I_{p}>0$. In the following, we approximate them by real values by setting $I_{p}=0$, arriving at

$$
\vec{k}+\vec{A}\left[t_{r}^{(i)}\right]=0 .
$$

Classical trajectories originating at different release times $t_{r}^{(i)}(i=1,2, \ldots)$ can give rise to semiclassical interferences provided they satisfy the condition given by Eq. (13) for reaching the same final momentum $\vec{k}$. While the interference condition involves the vector potential $\vec{A}$, the trajectory is governed by the electrical field $\vec{F}$. The precise relation between $\vec{F}$ and $\vec{A}$, in particular the constant of integration, is therefore of importance for few-cycle and multicycle pulses. We consider a laser field

$$
F(t)=f(t) \sin \left(\omega t+\phi_{\mathrm{CE}}\right),
$$

with $f(t)$ the envelope function and $\phi_{\mathrm{CE}}$ the carrier-envelope phase. The envelope function of the pulse should vanish as $t \rightarrow \pm \infty$. The vector potential is accordingly given by

$$
\begin{aligned}
A(t)= & -\int_{-\infty}^{t} d t^{\prime} F\left(t^{\prime}\right)=\frac{f(t)}{\omega} \cos \left(\omega t+\phi_{\mathrm{CE}}\right) \\
& -\frac{1}{\omega} \int_{-\infty}^{t} d t^{\prime} f^{\prime}\left(t^{\prime}\right) \cos \left(\omega t^{\prime}+\phi_{\mathrm{CE}}\right) .
\end{aligned}
$$

For a multicycle pulse we can assume a slowly varying envelope function on the time scale of the optical cycle [i.e. $\left.\left|f^{\prime}(t)\right| \ll \omega|f(t)|\right]$. Consequently,

$$
A(t) \simeq \frac{f(t)}{\omega} \cos \left(\omega t+\phi_{\mathrm{CE}}\right) .
$$

As an example of a few-cycle pulse, let us consider exactly one sinusoidal oscillation with $\phi_{\mathrm{CE}}=0$ within a rectangularshaped envelope

$$
f(t)=F_{0} \theta(\pi / \omega-t) \theta(\pi / \omega+t) .
$$

The vector potential becomes now

$$
A(t)=\frac{f(t)}{\omega}[\cos (\omega t)-1] .
$$

Unlike in the multicycle case with a slowly varying envelope function [Eq. (16)], the vector potential is in the singlecycle case [Eq. (18)] negative-definite, implying, within the semiclassical limit [Eq. (13)], unipolar electron emission in a preferred direction [12]. The latter is a consequence of the pronounced carrier-envelope -phase dependence and inversion symmetry breaking of near-single cycle pulses.

We now introduce an envelope function that, unlike Eq. (17), reproduces the intracycle interferences characteristic for multicycle cycles irrespective of the number of cycles the envelope covers. We use an $N$-cycle flat-top pulse with $m$ - and $m^{\prime}$-cycle linear ramp-on and ramp-off, respectively,

$$
f(t)=F_{0}\left\{\begin{array}{lll}
\left(\frac{\omega t}{2 \pi m}+1\right) & \text { if } \quad \frac{-2 m \pi}{\omega} \leqslant t<0 \\
1 & \text { if } \quad 0 \leqslant t<\frac{2 N \pi}{\omega} \\
\frac{2\left(N+m^{\prime}\right) \pi-\omega t}{2 \pi m^{\prime}} & \text { if } \quad \frac{2 N \pi}{\omega} \leqslant t<\frac{2\left(N+m^{\prime}\right) \pi}{\omega} .
\end{array}\right.
$$

The important point to note is that for $N$ an integer, $\phi_{\mathrm{CE}}=0$, and $m$ and $m^{\prime}$ integer or half-integer numbers, the vector potential in the flat-top region is given by Eq. (16). Note that good approximations to pulses similar to Eq. (19) can be experimentally produced by Fourier shaping [32]. 
With the vector potential of the form [Eq. (16)] with $\phi_{\mathrm{CE}}=0$, the semiclassical ionization times within the SMM [Eq. (13)] fulfills

$$
\cos \left(\omega t_{r}^{(i)}\right)=-\kappa,
$$

where

$$
\kappa=\frac{\omega k}{F_{0}},
$$

denotes the dimensionless scaled momentum. As we consider in the remainder of this section a 1D problem, $k$ is a scalar denoting the momentum along the direction of the laser polarization axis. For a given value of $\kappa$, the field strength for ionization is independent of the particular trajectory considered, $\left|F\left(t_{r}^{(i)}\right)\right|=F_{0} \sqrt{1-\kappa^{2}}$. Assuming now that the ground-state depletion is negligible, the ionization rate $\Gamma(k)=$ $\left|G\left[t_{r}^{(i)}, k\right]\right|^{2}$ is identical for all subsequent ionization bursts (or trajectories) and is only a function of the time-independent final momentum $k$. Consequently, the momentum distribution [Eq. (3)] can be written within the SMM as

$$
\frac{d P}{d k}^{\mathrm{SM}}=\Gamma(k)\left|\sum_{i=1}^{M} e^{i S^{\mathrm{SM}}\left[t_{r}^{(i)}\right]}\right|^{2},
$$

where the second factor on the right-hand side of Eq. (22) describes the interference of $M$ classical trajectories with final momentum $k$, where $t_{r}^{(i)}$ is a function of $k$ through Eq. (20).

From Eq. (7), the semiclassical action along one electron trajectory with release time $t_{r}^{(i)}$ is, up to a constant,

$$
\begin{aligned}
S^{\mathrm{SM}}\left[t_{r}^{(i)}\right]= & 2 U_{p}\left[\left(1+\frac{\cos \left(2 \omega t_{r}^{(i)}\right)}{2}\right) t_{r}^{(i)}-\frac{3}{4 \omega} \sin \left(2 \omega t_{r}^{(i)}\right)\right] \\
& +I_{p} t_{r}^{(i)},
\end{aligned}
$$

where the ponderomotive energy is given by $U_{p}=F_{0}^{2} / 4 \omega^{2}$. As there are two interfering trajectories per cycle, $M=2 N$, with $N$ being the number of cycles involved, the sum over interfering trajectories [Eq. (22)] can now be decomposed into those associated with two release times within the same cycle and those associated with release times in different cycles [18]

$$
\begin{aligned}
\sum_{i=1}^{2 N} e^{i S^{\mathrm{SM}}\left[t_{r}^{(i)}\right]} & =\sum_{j=1}^{N} \sum_{\alpha=1}^{2} e^{i S^{\mathrm{SM}}\left[t_{r}^{(j, \alpha)}\right]} \\
& =2 \sum_{j=1}^{N} e^{i \bar{S}_{j}^{\mathrm{SM}}} \cos \left(\frac{\Delta S_{j}^{\mathrm{SM}}}{2}\right),
\end{aligned}
$$

where $\bar{S}_{j}^{\mathrm{SM}}=\left[S^{\mathrm{SM}}\left[t_{r}^{(j, 1)}\right]+S^{\mathrm{SM}}\left[t_{r}^{(j, 2)}\right]\right] / 2$ is the average action of the two trajectories released in cycle $j$, and $\Delta S_{j}^{\mathrm{SM}}=$ $S^{\mathrm{SM}}\left[t_{r}^{(j, 1)}\right]-S^{\mathrm{SM}}\left[t_{r}^{(j, 2)}\right]$ is the accumulated action between the two release times $t_{r}^{(j, 1)}$ and $t_{r}^{(j, 2)}$ within the same $j$ th cycle. The underlying time structure is schematically illustrated in Fig. 1. There are two solutions of Eq. (20) per optical cycle: the early release time $t_{r}^{(j, 1)}$, within the first half of the $j$ th cycle (marked by circles in Fig. 1), and the late release time $t_{r}^{(j, 2)}$, within the second half of the $j$ th cycle (marked by triangles in

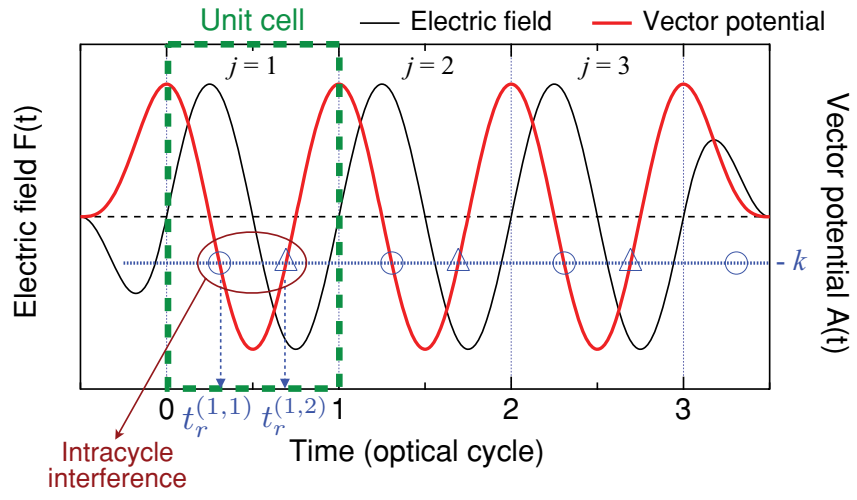

FIG. 1. (Color online) Electric field $F(t)$ (left axis) and vector potential $A(t)$ (right axis) of a flat-top pulse [as defined in Eq. (19) with $\left.N=3, m=m^{\prime}=1 / 2\right]$. The electron emission times for a given final momentum $k$ are marked by circles $\left[t_{r}^{(j, 1)}\right]$ and triangles $\left[t_{r}^{(j, 2)}\right]$. Each pair of circles and triangles determines the structure factor $F(k)$ and leads to intracycle interference while the periodic train of such pairs gives rise to intercycle interference. Each optical cycle can be viewed as a "unit cell" of the time lattice.

Fig. 1). Within the SMM, the average action depends linearly on the cycle number $j$

$$
\bar{S}_{j}^{\mathrm{SM}}=S_{0}+j \tilde{S},
$$

where $S_{0}$ is a constant which does not enter when taking the absolute value of Eq. (24) is taken, and $\tilde{S}=(2 \pi / \omega)\left(E+U_{p}+\right.$ $I_{p}$ ). In turn, the difference of the action $\Delta S_{j}^{S M}$ is a constant independent of the cycle number $j$, which can be expressed (dropping the subindex $j$ ) as

$$
\Delta S^{\mathrm{SM}}=\frac{2 U_{p}}{\omega}\left[\left(1+2 \kappa^{2}+2 \gamma^{2}\right) \arccos \kappa-3 \kappa \sqrt{1-\kappa^{2}}\right],
$$

where $\gamma=\sqrt{2 I_{p}} \omega / F_{0}$ is the Keldysh parameter.

After some algebra, Eq. (22) can be rewritten as

$$
\frac{d P}{d k}=4 \Gamma(k) \underbrace{\cos ^{2}\left(\frac{\Delta S^{\mathrm{SM}}}{2}\right)}_{F(k)} \underbrace{\left[\frac{\sin (N \tilde{S} / 2)}{\sin (\tilde{S} / 2)}\right]^{2}}_{B(k)} .
$$

Equation (27) indicates that the interference pattern can be factorized in two contributions: (i) the interference stemming from a pair of trajectories within the same cycle (intracycle interference), governed by $F(k)$ and (ii) the interference stemming from trajectories released at different cycles (intercycle interference) resulting in the well-known ATI peaks given by $B(k)$ (see Ref. [33]). The intracycle interference arises from the superposition of pairs of classical trajectories separated by a time slit $\Delta t=t_{r}^{(j, 1)}-t_{r}^{(j, 2)}$ of the order of less than half a period of the laser pulse (i.e., $\Delta t<\pi / \omega)$ giving access to emission time resolution of $\lesssim 1$ fs (for near infrared pulses), while the difference between $t_{r}^{(j, \alpha)}$ and $t_{r}^{(j+1, \alpha)}$ is $2 \pi / \omega$ (i.e., the optical period of the laser). Equation (27) is structurally equivalent to the intensity for crystal diffraction: The factor $F(k)$ represents the form (or structure) factor accounting for interference modulations due to the internal structure within the unit cell while the factor $B(k)$ gives rise to Bragg peaks 


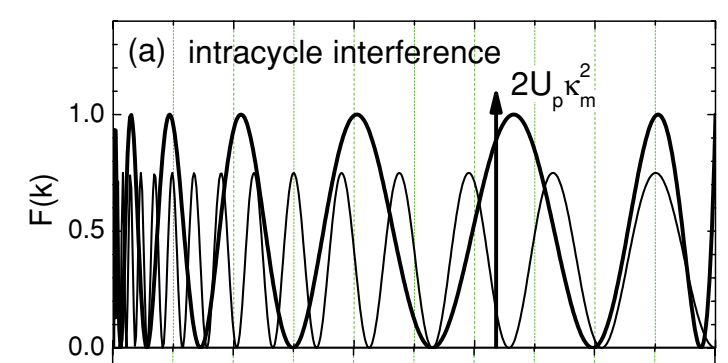

(b) intercycle interference

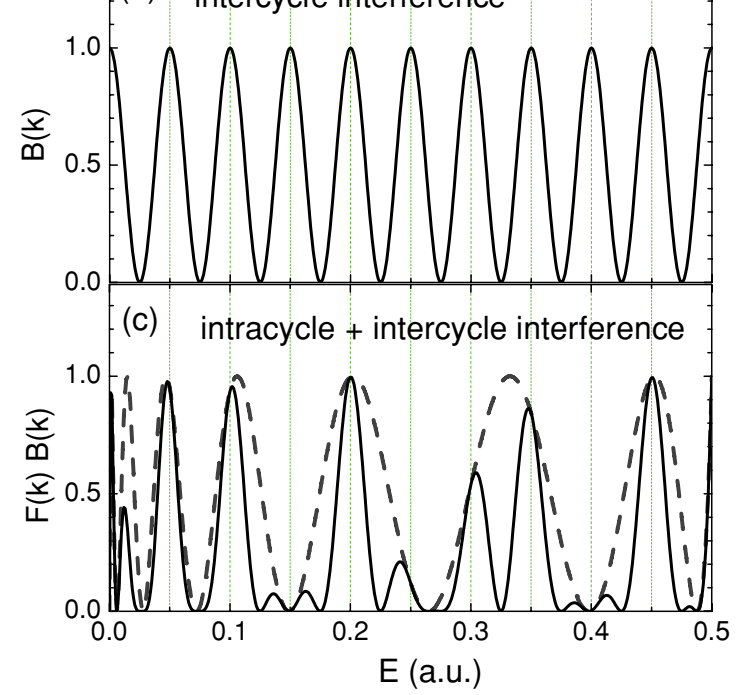

FIG. 2. (Color online) (a) Buildup of the interference pattern following the semiclassical SMM: intracycle interference pattern given by the structure pattern $F(k)$ for a multicycle pulse [Eq. (16)]. The thin line shows the pattern for a single-cycle pulse [Eq. (17)]. (b) Intercycle interference given by the function $B(k)$ with $N=2$. (c) Total interference pattern $F(k) B(k)$ [see Eq. (27)] with $N=2$. The laser parameters are $F_{0}=0.05, \omega=0.05$, and $I_{p}=0.5$. Vertical grid lines correspond to multiphoton energies.

due to the periodicity of the crystals. Alternatively, $B(k)$ in Eq. (27) may be viewed as a diffraction grating in the time domain consisting of $N$ slits where $F(k)$ is the diffraction factor for each slit.

We will analyze in the following how the interplay between $B(k)$ and $F(k)$ controls the spectrum of direct ATI electrons. The intracycle structure factor $F(k)$ [shown in Fig. 2(a)] displays oscillations with maxima unrelated to multiphoton peaks of spacing $\hbar \omega$ produced by the factor $B(k)$. The condition for intracycle interference peaks is $\Delta S^{\mathrm{SM}}=2 m \pi$. As $\Delta S^{S M}$ is a finite, monotonically decreasing function of $\kappa$ and $\Delta S^{\mathrm{SM}}=\pi R$ for $k=0$, therefore there is a well defined number of intracycle peaks in the spectral region of direct emission $\left(E<2 U_{p}\right)$. This number is equal to $n_{\text {intra }}=R / 2=$ $\left(U_{p}+I_{p}\right) / 2 \omega$. For the case of intercycle interference the number of ATI peaks in the same region is $n_{\text {inter }}=2 U_{p} / \omega$. Thus, the ratio of the number of intracycle peaks relative to those for intercycle peaks is "universal" as it depends only on the Keldysh parameter

$$
\frac{n_{\text {intra }}}{n_{\text {inter }}}=\frac{1}{4}+\frac{\gamma^{2}}{2} .
$$

Therefore, $n_{\text {intra }}$ is lower than $n_{\text {inter }}$ provided that $\gamma<\sqrt{3 / 2}$, in the tunneling regime and transition region to the multiphoton regimes. For ionization by long wavelengths $(\gamma \ll 1)$, the photoelectron spectrum will show, on average, four ATI peaks per intracycle peak which emphasizes the modulation of ATI peaks by the intracycle pattern. By contrast, for $\gamma>\sqrt{3 / 2}$, $n_{\text {intra }}$ can exceed $n_{\text {inter }}$ (i.e., the multiphoton peaks now modulates the intracycle interference pattern). It should be noted, however, that in this regime the SMM may break down.

For an arbitrary value of $N \geqslant 2$, Eq. (27) predicts consecutive multiphoton peaks at $E_{n}=n \hbar \omega-\left(I_{p}+U_{p}\right)$ with integer $n$, consistent with the absorption of $n$ photons. If $N \geqslant 3$, then $N-2$ secondary fringes produced by the interference of $N$ slits in a diffraction grating appear. In multicycle photoelectron spectra with $N \geqslant 2$, both intracycle and intercycle interferences are simultaneously present. For the simplest case of $N=2$, the factor $B(k)$ reduces to the two-slit Young interference expression

$$
B(k)=4 \cos ^{2}\left[\pi / \omega\left(E+U_{p}+I_{p}\right)\right],
$$

shown in Fig. 2(b). When $N \rightarrow \infty$, the factor $B(k)$ becomes a sequence of delta functions situated at $E_{n}$. The separation of consecutive peaks of the intracycle factor $F(k)$ depends on energy in a nontrivial way. In Fig. 2(c) we show the interference pattern [Eq. (27)] for $N=2$. To focus on the interference process, only the factor $F(k) B(k)$ normalized to unity is displayed, disregarding the variation of the ionization rate $\Gamma(k)$. The multiphoton peaks given by $B(k)[$ Fig. 2(b)] are modulated by the intracycle interference factor $F(k)$. Intracycle interference can lead to slight shifts of the multiphoton peaks as observed in Fig. 2(c) relative to Fig. 2(b) for the peaks at $E=0.25$ (slightly shifted to the left) and $E=0.3$ (slightly shifted to the right), and even to the suppression of multiphoton peaks (near $E=0.15$ and $E=0.4$ ). The separation of consecutive peaks of the intracycle envelope factor $F(k)$ is higher at intermediate energies than near the classical boundaries $E=0$ and $E=2 U_{p}=0.5$. This leads to another effect near threshold: the splitting of the multiphoton peaks, as observed in Fig. 2(c) for the peak at $E_{n}=0$ (with $n=15)$.

\section{B. Frequency dependence}

We now proceed by studying the dependence of the photoelectron spectrum on the carrier frequency of the laser field. It is convenient to present the energy and frequency dependence of the spectrum in terms of the channel-closing number $R$ defined in Eq. (1) and the continuous scaled energy $n^{*}$ defined as

$$
n^{*}=\frac{E}{\hbar \omega}+R,
$$

instead of $\omega$ and $E$, respectively. $R$ corresponds to the minimum number of absorbed photons required for ionization and the real number $n^{*}$ coincides with the ATI order at integer values $\left(n^{*}=n\right)$. It has been recently shown that the yield of HHG and high-order ATI (the latter in the rescattering region) oscillates with driver intensity and wavelength with a period of unity in terms of $R[1,6,8]$. In Fig. 3 we show the interference pattern contained in $F(k) B(k)$ as a function 

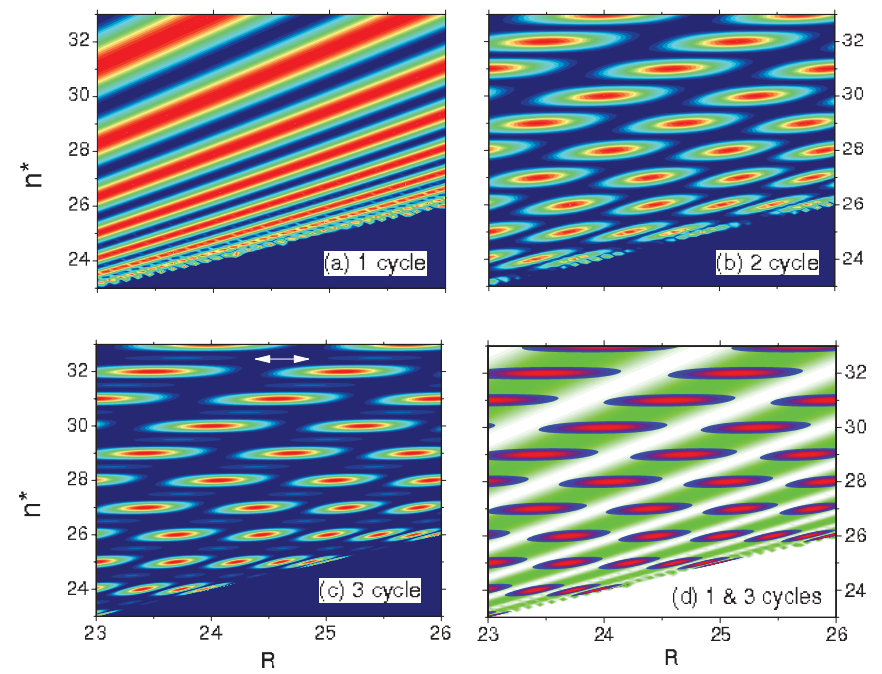

FIG. 3. (Color online) Semiclassical total ionization probability within the SMM as a function of the channel-closing parameter $R$ and the scaled energy $n^{*}$ (see text) calculated for (a) $N=1$, (b) $N=2$, and (c) $N=3$. In (d), the results of (a) (green stripes) with those of (c) are overlaid (blue-red islands). $F_{0}=0.0675$ (intensity equal to $1.6 \times 10^{14} \mathrm{~W} / \mathrm{cm}^{2}$ ) and $I_{p}=0.5$. The range in $R$ corresponds to wavelengths $1000-1060 \mathrm{~nm}$ or frequencies $0.0456-0.0430$ a.u.. The white arrow in (c) points to one secondary fringe.

of $n^{*}$ and $R$ in terms of a two-dimensional interferogram. For $N=1$, we observe that the intracycle interference $F(k)$ results in a family of oblique stripes which broaden as $n^{*}$ increases [Fig. 3(a)]. As there is only one cycle involved, intercycle interference is absent $[B(k)=1]$. For $N=2$ [Fig. 3(b)], two types of interference emerge: intracycle interference as oblique stripes and intercycle interference as horizontal stripes situated about integer values of $n^{*}$. The former, described by $F(k)$ of Eq. (27), is due to the interference of the two pairs of trajectories $\left[t^{(1,1)}, t^{(1,2)}\right]$ and $\left[t^{(2,1)}, t^{(2,2)}\right]$ each within the same optical cycle. The latter, described by factor $B(k)$ in Eq. (27), stems from the interference of classical trajectories of electrons detached at different optical cycles with a delay of $2 \pi / \omega$. The intercycle horizontal stripes centered at energy $E_{n}=(n-R) \omega$ with integer $n$ correspond to the well-known ATI-peaks. The horizontal ATI stripes are now superimposed on the intracycle interference pattern of Fig. 2(a), leading to a modulation of the ATI horizontal iso-energy stripes with the oblique stripe pattern [Eq. (27)]. In a similar two-dimensional (2D) interferogram for $N=3$ [Fig. 3(c)], the intercycle horizontal stripes are narrower than those observed for two cycles in Fig. 3(b) since the pulse is longer. In addition, one weak secondary fringe can be also observed between two consecutive ATI fringes. By contrast, the width of the intracycle oblique stripes are independent of the number of laser cycles since the time slit within one cycle remains unaltered irrespective of the number of cycles considered. This fact is highlighted in Fig. 3(d), where the oblique fringes due to intracycle interference [Fig. 3(a)] are overlaid on the horizontal ATI stripes [Fig. 3(c)]. We observe that the spots are located exactly on the intracycle oblique stripes, demonstrating that the intracycle interference is as important as the intercycle interference for the overall shape of the energy distribution.

It is interesting to contrast the intracycle interference pattern resulting from a multicycle inversion symmetry-of the $k_{z}$ distribution-preserving pulse [Eq. (19)] with the double-slit interference resulting from a single-cycle pulse with a vector potential of the form [Eq. (18)] described in Ref. [12]. This gives rise to a very different interference pattern, also shown in Fig. 2(a). In general, a variety of interference patterns can be produced by few-cycle pulses depending on the envelope and carrier-envelope phase.

\section{Spacing of intracycle peaks}

We analyze now the structure factor for intracycle interference $F(k)$ in more detail. To determine the separation between consecutive intracycle peaks, the variation of the action [Eq. (26)] can be expressed as $\Delta S(E+\Delta E) \simeq$ $\Delta S(E)+\frac{d \Delta S(E)}{d E} \Delta E$, where $\frac{d \Delta S(E)}{d E}$ plays the role of an energydependent (or chirped) frequency. The energy-dependent period $\Omega(E)$ of the interference oscillation, namely the separation of two consecutive peaks, can be expressed as a continuous function of the energy

$$
\Omega(E)=\frac{2 \pi}{\left|\frac{d \Delta S(E)}{d E}\right|} .
$$

After some algebraic manipulations, $\Omega(E)$ can be rewritten as a function of the dimensionless scaled momentum $\kappa$ as

$$
\Omega(\kappa)=\frac{\omega \pi \kappa \sqrt{1-\kappa^{2}}}{1-\kappa^{2}+\gamma^{2} / 2-\kappa \sqrt{1-\kappa^{2}} \arccos \kappa} .
$$

Equation (32) shows that the period of intracycle interference normalized to the photon energy $\Omega(\kappa) / \omega$, as a function of $\kappa$, depends on the laser parameters only through $\gamma$. In Fig. 4(a) we show $\Omega(\kappa) / \omega$ for different values of $\gamma$ in the multiphoton, transition, and tunneling regimes. In both the tunneling and transition regimes (i.e., $\gamma \lesssim 1$ ) the period of intracycle interference pattern is of the same order of magnitude as the photon energy $\hbar \omega$ over a wide range of the scaled momenta $\kappa$. In particular, in the tunneling limit $(\gamma \ll 1)$, both periods coincide near $\kappa \simeq 0.22,\left(E \simeq 0.097 U_{p}\right)$. By contrast, in the deep multiphoton regime $(\gamma \gg 1)$ the period of intracycle interference is several orders of magnitude smaller than the multiphoton peak separation (i.e., $\Omega(\kappa) \ll \omega$ ) which would preclude the experimental observation of intracycle peaks. However, the model is questionable in this regime.

One interesting observation is that the intracycle period is not a monotonic function of $\kappa$ (or $E$ ) but it possesses a maximum. The maximum of $\Omega(\kappa)$ appears at

$$
\kappa_{m}=\sqrt{1+\frac{\gamma^{2}}{2}-\frac{\gamma}{2} \sqrt{2+\gamma^{2}}} .
$$

In the tunneling limit $(\gamma \rightarrow 0), \kappa_{m}=1$, or equivalently, $E_{m}=2 U_{p}$, which corresponds to the classical cutoff for direct electrons. In the multiphoton limit $(\gamma \rightarrow \infty) \kappa_{m}=1 / \sqrt{2}$, which corresponds to $E_{m}=U_{p}$, just half the classical cutoff energy $2 U_{p}$. In general, $\kappa_{m}$ lies between these two limiting values [Fig. 4(b)]. To illustrate the applicably of Eq. (33) we 

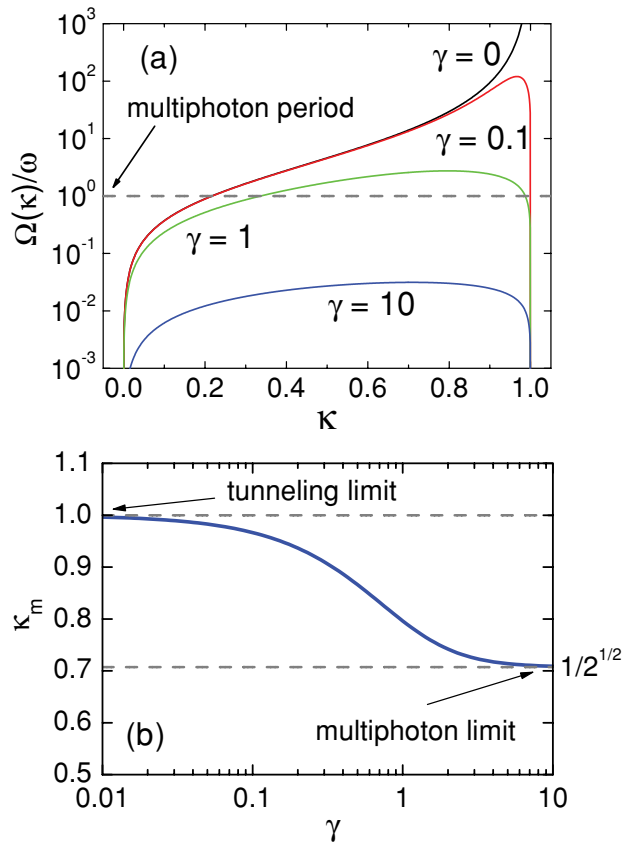

FIG. 4. (Color online) (a) Semiclassical prediction [Eq. (32)] for the separation between consecutive intracycle interference peaks (divided by the laser frequency) as a function of the scaled momentum $\kappa$, for $\gamma=0,0.1,1$, and 10; the horizontal dashed line corresponds to the scaled period of the multiphoton peaks. (b) Maximum $\kappa_{m}$ as a function of the Keldysh parameter $\gamma$ [see Eq. (33)]. The tunneling and multiphoton limits are marked with dashed lines.

have indicated the energy position corresponding to $\kappa_{m}$ in Fig. 2(a) by a vertical arrow [Fig. 4(b)].

\section{Influence of the Coulomb field}

The time grating picture emerging from the semiclassical approximation within the SMM neglects the influence of the Coulomb field on the ionized electron. Before subjecting the present model to a detailed quantitative test against full and approximate quantum calculations, it is instructive to inquire within a semiclassical approximation to which extent longrange Coulomb interactions in the exit channel are expected to modify the interference picture. As a point of reference, we note that for HHG the Coulomb distortion gives rise to a shift of the spectrum relative to integer values of $R$ (see [4]). Our starting point is the inclusion of the Coulomb potential into the semiclassical action [Eq. (7)],

$$
S\left(t^{\prime}, t\right)=-\int_{t^{\prime}}^{t} d t^{\prime \prime}\left[\frac{\left[\vec{k}+\vec{A}\left(t^{\prime \prime}\right)\right]^{2}}{2}+V\left(r\left(t^{\prime \prime}, t^{\prime}\right)\right)+I_{p}\right],
$$

where $r\left(t^{\prime \prime}, t^{\prime}\right)$ is the trajectory of the electron released at $t^{\prime}$. Note that the Coulomb interaction is of the same order as $I_{p}$. Therefore, the real emission times $t_{r}^{(i)}$ determined from the extrema of the action [Eq. (13)] remain, to the same order of approximation, unchanged. However, the action determining the interference pattern will be modified. The correction to the action of Eq. (26) is

$$
S_{c}\left(t_{r}\right)=-\int_{t_{r}}^{\infty} V\left(r\left(t^{\prime}, t_{r}\right)\right) d t^{\prime},
$$

where the trajectory $r\left(t^{\prime}, t_{r}\right)$ is calculated within the SMM ignoring the Coulomb potential. Note that for an infinite pulse, the correction $S_{c}\left(t_{r}\right)$ to the SMM action within one cycle is independent of the cycle number $j$ the trajectory was born in. Consequently, the presence of the Coulomb potential neither destroys the factorization into intercycle and intracycle interferences [Eq. (27)] nor changes the intercycle interference pattern (position of the ATI peaks). The result for a finite number of cycles rapidly converge toward the infinite case since most trajectories rapidly leave the region near the nucleus. Even trajectories passing $z=0$ several times converge rapidly with the number of cycles if one includes the spreading of the initial wave packet into the consideration.

The presence of the Coulomb field will, however, change the intracycle phase as of the form factor $F(k)$. A displacement of the whole intracycle interference pattern of the photoelectron spectrum weakly dependent on the electron energy is obtained. We have used different soft potentials (for example the one in [4]) to mimic three-dimensional (3D) calculations employing the eikonal approximation. All of them agree qualitatively with quantum calculations (see below).

\section{TESTING THE SEMICLASSICAL MODEL}

To test the predictions of the 1D semiclassical models, we perform 3D quantum calculations employing the SFA, CVA, and TDSE methods for identical laser field parameters.

\section{A. Multicycle pulses}

We have calculated the 2D interferogram of ionization probabilities within the SFA and CVA for $N=3$ and $m=$ $m^{\prime}=1 / 2$, corresponding to the pulse in Fig. 1 . To explore the interferences, which appear along the laser polarization axis the SFA [Eqs. (5) and (6)] and CVA [Eqs. (5) and (8)] ionization probability of electrons ejected within a cone of $10^{\circ}$ around the $\pm z$ direction are shown (Fig. 5). As in the semiclassical model [Fig. 2(c)], the horizontal intercycle interference stripes at fixed values of ATI order $n$ are modulated by the oblique stripes stemming from the intracycle interference. This shows that the semiclassical model in Sec. II captures the essence of the interferences in the photoionization process. Comparing the SFA [Fig. 5(a)] with the CVA [Fig. 5(b)] interferogram, we observe a shift of the positions of the intracycle stripes. This shift is due to the presence of the Coulomb potential of the core, approximately accounted for in CVA but completely neglected in SFA (as in the SMM). This is consistent with our previous investigation using screened Coulomb potentials [18]. In addition, the positions of intercycle (ATI) interference horizontal stripes are not affected by the interaction of the remaining core with the receding electron and stay near integer values of $n^{*}$. The comparison between the SFA and the CVA allows for a direct probe of the effect of the Coulomb phase shift on the interference pattern in qualitative agreement 


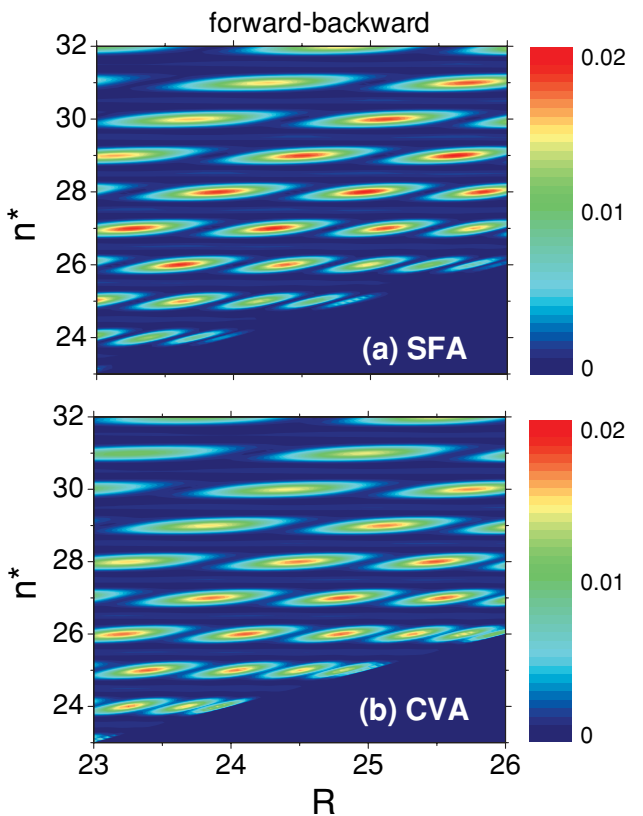

FIG. 5. (Color online) 2D interferogram for the ionization probability within a cone of $10^{\circ}$ in the forward and backward direction as a function of channel-closing parameter $R$ and scaled energy $n^{*}$ (see text), for a four-cycle pulse with $F_{0}=0.0675, N=3$ and with $m=m^{\prime}=1 / 2$, within the (a) SFA and (b) CVA.

with semiclassical correction of Eq. (35). By varying $Z_{T}$ in the final-state Coulomb distortion factor [Eq. (8)], the Coulomb phase can be varied between zero and its full value without affecting the initial-state binding energy or wave function. In Fig. 6, the photoelectron spectrum for the same parameters as in Fig. 5, but fix frequency $\omega=0.0456(R=$ 23 ), undergoes a monotonic shift of the intracycle envelope $F(k)$ as the strength of the Coulomb potential parametrized by the nuclear charge increases from $Z_{T}=0$ (SFA) to $Z_{T}=1$ (CVA) clearly illustrating the effect of the Coulomb tail on the form factor $F(k)$. Note that no significant changes of the multiphoton positions described by $B(k)$ are observed. A qualitatively similar result can be found using the 1D semiclassical model corrected for the approximate Coulomb phase presented in Sec. III D. The horizontal arrow in Fig. 6 indicates the shift in energy from the Coulomb correction to the SMM [Eq. (8)].

It is now of interest to compare the 1D semiclassical model and 3D approximate quantum description (CVA) with the exact solution of the TDSE (Fig. 7). The close similarity of the exact TDSE interferograms with the semiclassical results (Fig. 3), and the SFA and CVA (Fig. 5), underscores that the models catch the main characteristics of the interferences in the ionization processes by short laser pulses. In particular, the position and shape of the oblique stripes agree almost perfectly with the CVA. In Fig. 7(b), we show the TDSE interferogram when integrating over all angles. Since the doubly differential momentum distribution for high energies is localized close to the $k_{z}$ axis $[18,34,35]$, the full spectra decrease much more rapidly with energy than the spectra in the $10^{\circ}$ cone. Still some remainders of the interferences are visible in the angle-integrated spectra.

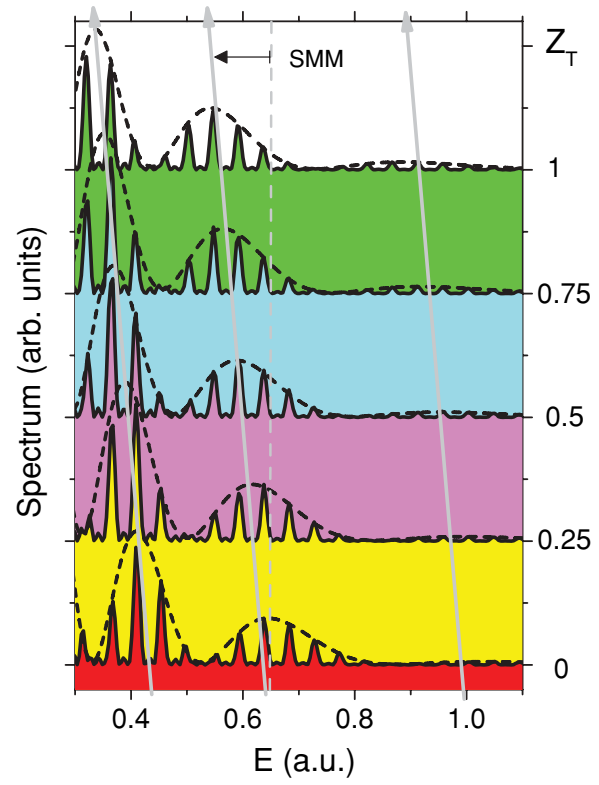

FIG. 6. (Color online) Photoelectron spectra for different values of the nuclear charge $Z_{T}=0,0.25,0.5,0.75$, and 1 within the CVA, from bottom to top calculated for the four-cycle pulse described in Fig. 5 (solid line) and the one-and-half cycle of Fig. 8 (dashed line). The carrier frequency is $\omega=0.0456(R=23)$. The horizontal arrow indicates the Coulomb shift estimated within the SMM.

While the flat-top pulse shape [Eq. (19)] is optimally suited to illustrate the interplay between intracycle and intercycle interferences, qualitatively similar patterns have been found for a sine-squared envelope. This indicates that the intracycle
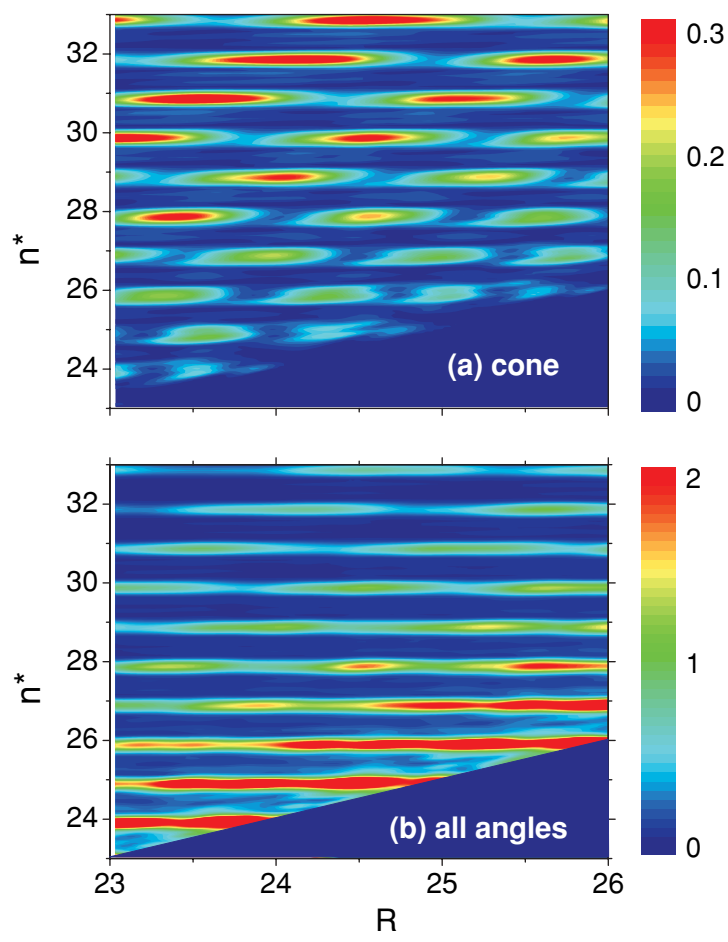

FIG. 7. (Color online) 2D TDSE interferogram for electron emission of hydrogen for the same field as in Fig. 5. (a) Into a cone of $10^{\circ}$ around the polarization axis and (b) into all angles. 

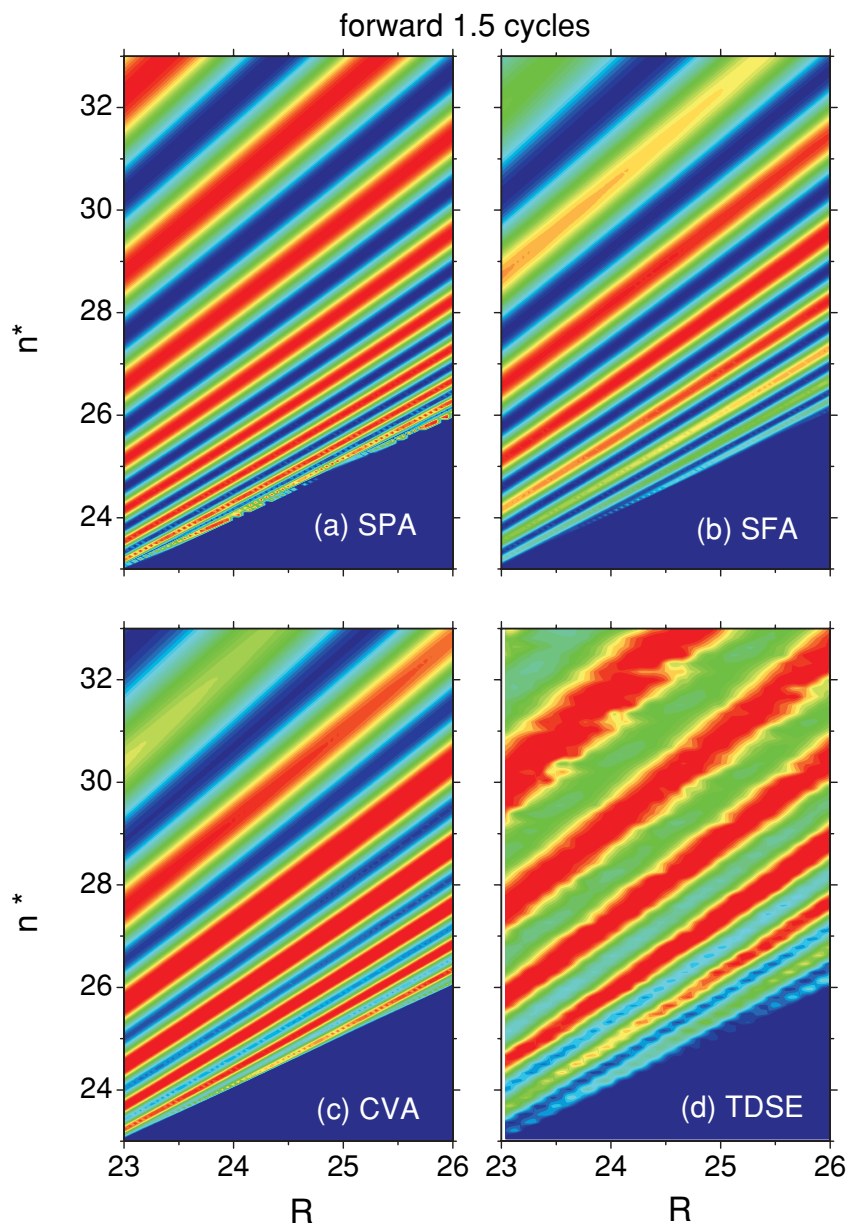

FIG. 8. (Color online) 2D interferogram for emission within a cone of $10^{\circ}$ around the polarization axis as a function of channelclosing parameter $R$ and scaled energy $n^{*}$ calculated for a 1.5-cycle pulse with $F_{0}=0.0675, m=0$, and $m^{\prime}=1 / 2$. (a) SPA, (b) SFA, (c) CVA, and (d) TDSE (see text).

modulations of the photoelectron spectra persist also for other pulse shapes, though the details might change.

\section{B. Isolating intracycle interference}

For the multicycle pulses considered so far, intracycle interference appears as a modulation envelope of discrete ATI peaks. To clearly illustrate intracycle interferences separately from intercycle ones we use almost single cycle pulses such that the vector potential fulfills Eq. (16) in the main region of the pulse. We consider a single-cycle $(N=1)$ pulse with a $m^{\prime}=1 / 2$ ramp off. The present results are without ramp on $(m=0)$, however, using $m=1 / 2$ does not significantly alter the results. The yield integrated within a cone of $10^{\circ}$ in the forward direction displays an intracycle interference pattern for SFA, CVA, and TDSE [Figs. 8(a), 8(b), and 8(c), respectively], similar to the prediction of the semiclassical model of Fig. 3(a). The position of the intracycle interference bands in Fig. 8(b) calculated within the SFA are close to the semiclassical SMM prediction [Fig. 3(a)]. The origin of the small deviations lies in that the complex saddle-point solutions for complex trajectories [Eq. (12)] have been approximated

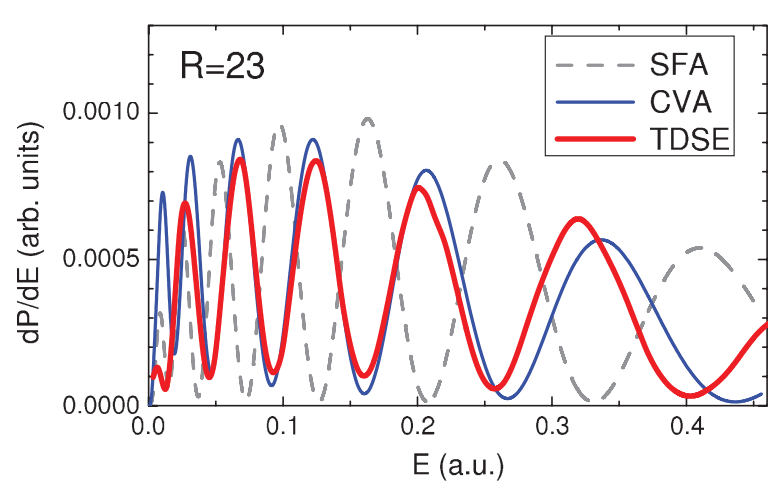

FIG. 9. (Color online) Energy distribution within a cone of $10^{\circ}$ around the polarization axis for the same laser parameters as in Fig. 8 for $\omega=0.0456(R=23)$. The data are from the SFA (dashed line), the CVA (thin solid line), and TDSE (thick solid line).

by the real solutions (classical trajectories) of Eq. (13) in the SMM. Using instead complex solutions of the stationary phase approximation (SPA) [Fig. 8(a)] gives an excellent agreement with the SFA [Fig. 8(b)]. A direct comparison of Figs. 8 with 5 confirms the invariance of the intracycle interference pattern with respect to the number of cycles involved. As mentioned previously, the shift between SFA results in Fig. 8(b) and CVA in Fig. 8(c) unveils the effect of the Coulomb potential in the dynamics of exiting electrons.

A more detailed quantitative comparison is presented in Fig. 9 in terms of a vertical cut through the distributions of Fig. 8 at $R=23(\omega=0.0456)$. The CVA photoelectron spectrum resembles quite accurately the TDSE energy distribution. The shift in the position between the SFA and CVA intracycle interference highlights the pattern shift of the intracycle interference by the Coulomb potential of the core. The Coulomb tail shifts the interference maxima toward lower energies, consistent with the results of Fig. 6.

In the following we compute the spacing of two consecutive intracycle peaks, $\Delta E$, of the photoelectron spectrum for $R=23$ as a function of the energy position of the peaks (Fig. 10). The agreement among the SFA, the CVA, and

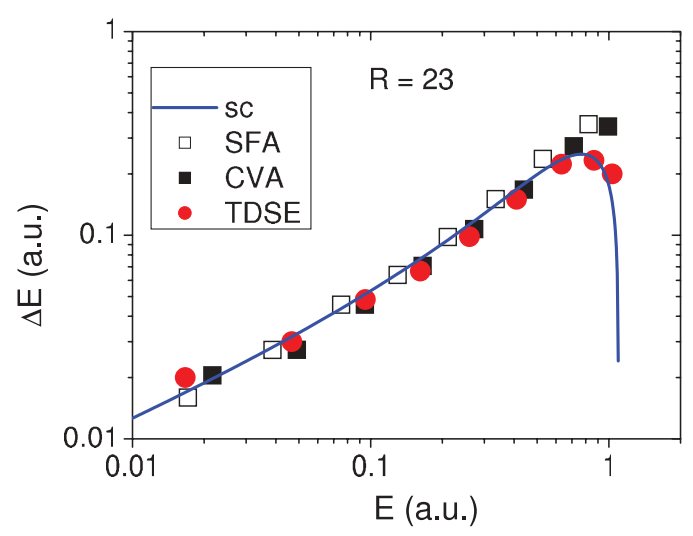

FIG. 10. (Color online) Energy difference between consecutive intracycle peaks as a function of the energy position of the corresponding peaks calculated with SFA (open squares), CVA (full squares), and TDSE (circles). The full line is the semiclassical prediction of Eq. (32). Same laser parameters as in Fig. 9. 


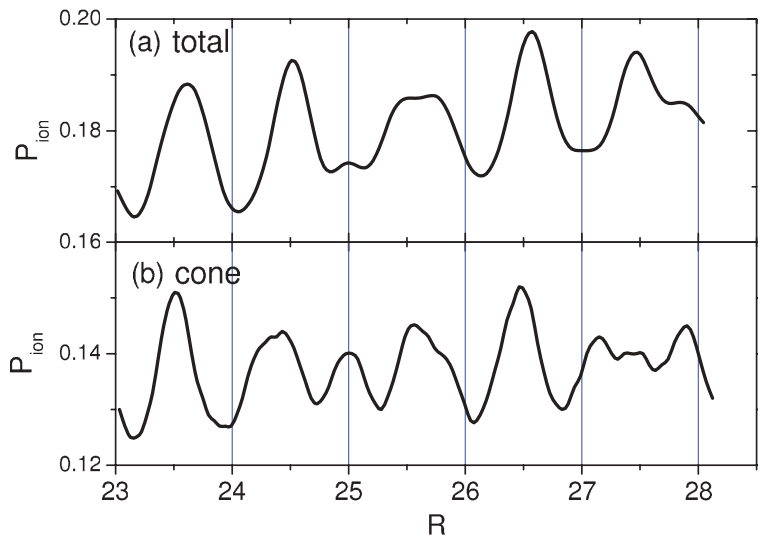

FIG. 11. (Color online) (a) TDSE total ionization probability $P_{\text {ion }}$ as a function of the channel-closing parameter $R$ (wavelengths in the range 1000 to $1100 \mathrm{~nm}$ ) calculated within the TDSE. The parameters of the laser field are $F_{0}=0.0675$ a.u. and the total number of cycles is $N=3$ with $m=m^{\prime}=1 / 2$ cycle for the ramp on and off. (b) Same as for (a) but for emission in a cone of $10^{\circ}$ around the polarization axis.

the TDSE is very good. We also include the semiclassical expression $\Omega(E)$ of Eqs. (31) and (32), which almost perfectly reproduces the quantum results. Even the predicted maximum at $E_{m}=\left(4 U_{p}\right) \kappa_{m}^{2} / 2=0.75$ [see Eq. (33)] is reproduced to some extent by the TDSE calculation. We also checked $\Delta E$ within the TDSE for $\omega=0.051$ (not shown) and found equally good results.

\section{Energy-integrated yield}

Finally, we consider the total ionization yield integrated over the photoelectron energy (i.e., along vertical lines of the interferograms) as a function of the channel-closing number. The total ionization probability calculated within the TDSE exhibits oscillations with a peak separation of unity in channel-closing number $R$ [see Fig. 11(a)]. Integer values of $R$ can be traced to the intersection of the horizontal multiphoton absorption lines with the diagonal marking the threshold for photoemission in Fig. 7(b). Depending on how rapidly the intensity along each vertical line decreases and on the details of the intracycle interferences, the maxima in the total yield are shifted to values slightly below the integer values of $R$ as seen in Fig. 11(a). The amplitude of oscillations of the ionization probability is about $4 \%$ of the average. We have confirmed that the periodicity $\Delta R=1$ holds accurately over the wavelength range between 800 and $2000 \mathrm{~nm}$ (figure not shown). These oscillations closely resemble those previously observed for the wavelength dependence of HHG $[20,22,24]$.

Focusing now on the ionization probability into a cone around the polarization axis [see Fig. 7(a)], a wide range of $n^{*}$ significantly contributes, as opposed to the threshold region for the total yield, resulting in a more complicated modulation pattern due to intracycle interferences. We show the yield for a $10^{\circ}$ cone in Fig. 11(b) as a function of $R$. Now the number of maxima and their distances are no longer close to $\Delta R=1$. The number and positions of the maxima at small angles are strongly varying with the angle (figure not shown). With increasing angle, the position of the maxima shift and the number of recognizable maxima decrease. Finally, integrating over the full angle, the rule $\Delta R=1$ is recovered. The reason for this angle dependence is that for energyintegrated yield within a cone of small angles around the polarization axis, remnants of intracycle interferences are visible, while in the total yield only intercycle interferences can be observed.

\section{CONCLUSION}

In this article we have presented a study of interference effects observed in the direct atomic ionization spectrum resulting from high-intensity multicycle laser pulses. We have identified the interplay between the intracycle and intercycle interferences of electron trajectories in photoelectron spectra by multicycle laser pulses. Intercycle interference corresponds to the well-known ATI peaks of the photoelectron spectrum arising from the superposition of wave packets released during different optical cycles, whereas intracycle interference comes from the coherent superposition of electron wave packets released within the same optical cycle. 2D interferograms as a function of the channel-closing number $R$ and the scaled energy $n^{*}$ are calculated within the SFA, the CVA, and the TDSE. The intercycle interference pattern is displayed as horizontal stripes located at integer values of ATI order $n$ modulated by the intracycle interference pattern observed as oblique stripes. The intracycle interference modulation can be most clearly seen for emission in the direction of the laser-polarization axis and is independent of the total number of optical cycles involved in the laser pulse. We present a simple semiclassical model based on the SMM that explains successfully the calculated patterns. An analytical expression for the complete interference pattern and the separation of intracycle peaks is presented showing an excellent agreement with the numerical calculations. The intracycle modulations are dependent on the long-range atomic Coulomb potential. This might open the possibility of imaging the core potential in the experimentally easy to obtain direct electron yield. Since intracycle interferences result from pairs of emission points closely spaced in time, the spacing between adjacent interference maxima in the spectrum for energies above a small fraction of the ponderomotive energy is wider than the spacings of the ATI peaks. Therefore, intracycle interferences should be recognizable even in low-resolution spectra. Carefully tailoring the field, the intracycle interferences may be experimentally observable also for almost-single cycle pulses.

\section{ACKNOWLEDGMENTS}

This work was supported by CONICET and PICT200600772 of ANPCyT (Argentina), UBACyT X147, SFB 016 ADLIS and P15025-N08 of FWF (Austria). K.L.I. gratefully acknowledges financial support by the JST-PREST program, the MEXT Grant No. 19686006 (Japan), and the Advanced Photon Science Alliance (APSA) project (Japan) and K.S. acknowledges additional support by the IMPRS program of the MPQ (Germany). 
[1] K. Schiessl, K. L. Ishikawa, E. Persson, and J. Burgdörfer, Phys. Rev. Lett. 99, 253903 (2007); J. Mod. Opt. 55, 2617 (2008).

[2] M. V. Frolov, N. L. Manakov, and A. F. Starace, Phys. Rev. Lett. 100, 173001 (2008).

[3] D. B. Milošević, E. Hasović, S. Odžak, M. Busuladžić, A. Gazibegović-Busuladžić, and W. Becker, J. Mod. Opt. 55, 2653 (2008).

[4] K. L. Ishikawa, K. Schiessl, E. Persson, and J. Burgdörfer, Phys. Rev. A 79, 033411 (2009).

[5] B. Borca, A. F. Starace, A. V. Flegel, M. V. Frolov, and N. L. Manakov, Phys. Rev. A 65, 051402(R) (2002).

[6] D. B. Milošević and W. Becker, Phys. Rev. A 66, 063417 (2002).

[7] P. B. Corkum, N. H. Burnett, and M. Y. Ivanov, Opt. Lett. 19, 1870 (1994).

[8] D. B. Milošević, E. Hasovic, M. Busuladzic, A. GazibegovicBusuladzic, and W. Becker, Phys. Rev. A 76, 053410 (2007).

[9] C. P. J. Martiny and L. B. Madsen, Phys. Rev. A 76, 043416 (2007).

[10] F. Lindner, M. G. Schätzel, H. Walther, A. Baltuška, E. Goulielmakis, F. Krausz, D. B. Milošević, D. Bauer, W. Becker, and G. G. Paulus, Phys. Rev. Lett. 95, 040401 (2005).

[11] R. Gopal et al., Phys. Rev. Lett. 103, 053001 (2009).

[12] D. G. Arbó, E. Persson, and J. Burgdörfer, Phys. Rev. A 74, 063407 (2006); D. G. Arbó, S. Yoshida, E. Persson, K. I. Dimitriou, and J. Burgdörfer, J. Phys.: Conferences Series 88, 012054 (2007).

[13] L. Guo, S. S. Han, and J. Chen, Opt. Express 18, 1240 (2010).

[14] D. G. Arbó, S. Yoshida, E. Persson, K. I. Dimitriou, and J. Burgdörfer, Phys. Rev. Lett. 96, 143003 (2006); D. G. Arbó, K. I. Dimitriou, E. Persson, and J. Burgdörfer, Phys. Rev. A 78, 013406 (2008).

[15] T. Marchenko, H. G. Muller, K. J. Schafer, and M. J. J. Vrakking, J. Phys. B 43, 095601 (2010).

[16] B. Bergues, Z. Ansari, D. Hanstorp, and I. Y. Kiyan, Phys. Rev. A 75, 063415 (2007).

[17] S. Bivona, G. Bonanno, R. Burlon, D. Gurrera, and C. Leone, Phys. Rev. A 77, 051404 (2008); S. Bivona, G. Bonanno, R. Burlon, and C. Leone, ibid. 79, 035403 (2009).
[18] D. G. Arbó, K. L. Ishikawa, K. Schiessl, E. Persson, and J. Burgdörfer, Phys. Rev. A 81, 021403 (2010).

[19] A. K. Kazansky and N. M. Kabachnik, J. Phys. B 43, 035601 (2010).

[20] P. A. Macri, J. E. Miraglia, and M. S. Gravielle, J. Opt. Soc. Am. B 20, 1801 (2003).

[21] V. D. Rodriguez, E. Cormier, and R. Gayet, Phys. Rev. A 69, 053402 (2004); V. D. Rodriguez, P. A. Macri, and D. G. Arbó, Nucl. Instrum. Methods B 267, 334 (2009).

[22] M. Lewenstein, K. C. Kulander, K. J. Schafer, and P. H. Bucksbaum, Phys. Rev. A 51, 1495 (1995); M. Lewenstein, Ph. Balcou, M. Yu. Ivanov, A. L'Huillier, and P. B. Corkum, ibid. 49, 2117 (1994).

[23] C. C. Chirila and R. M. Potvliege, Phys. Rev. A 71, 021402(R) (2005).

[24] M. Ivanov, P. B. Corkum, T. Zuo, and A. Bandrauk, Phys. Rev. Lett. 74, 2933 (1995).

[25] D. P. Dewangan and J. Eichler, Phys. Rep. 247, 59 (1997).

[26] D. M. Volkov, Z. Phys. 94, 250 (1935).

[27] D. G. Arbó, J. E. Miraglia, M. S. Gravielle, K. Schiessl, E. Persson, and J. Burgdörfer, Phys. Rev. A 77, 013401 (2008).

[28] G. Duchateau, E. Cormier, and R. Gayet, Eur. Phys. J. D 11, 191 (2000); G. Duchateau, E. Cormier, H. Bachau, and R. Gayet, Phys. Rev. A 63, 053411 (2001).

[29] X.-M. Tong and S. Chu, Chem. Phys. 217, 119 (1997).

[30] T. N. Rescigno and C. W. McCurdy, Phys. Rev. A 62, 032706 (2000).

[31] B. I. Schneider and L. A. Collins, J. Non-Cryst. Solids 351, 1551 (2005).

[32] T. Brixner and G. Gerber, Phys. Chem. Phys. Chem. 4, 418 (2003); P. Nuernberger, G. Vogt, T. Brixner, and G. Gerber, Phys. Chem. Chem. Phys. 9, 2470 (2007).

[33] F. H. M. Faisal and G. Schlegel, J. Phys. B 38, L223 (2005).

[34] A. Rudenko, K. Zrost, C. D. Schröter, V. L. B. de Jesus, B. Feuerstein, R. Moshammer, and J. Ullrich, J. Phys. B 37, L407 (2004).

[35] C. M. Maharjan, A. S. Alnaser, I. Litvinyuk, P. Ranitovic, and C. L. Cocke, J. Phys. B 39, 1955 (2006). 